\title{
Cortical evoked responses to synonyms and antonyms
}

\author{
JONATHAN VAUGHAN, KATHY SHERIF, RICHARD L. O'SULLIVAN, \\ DOUGLAS J. HERRMANN, and DOUGLAS A. WELDON \\ Hamilton College, Clinton, New York 13323
}

\begin{abstract}
The brain's processing of synonymity and antonymy was explored by examining the cortical evoked responses to correct judgments that a test word was a synonym or an antonym of a standard word presented 1 sec previously. Each of five subjects judged 256 pairs of words in each of two sessions. The evoked response to the second word was averaged separately for synonym and antonym pairs. Presentation of each test word as a synonym or an antonym, the order of presentation of each pair, and the side of the "synonym" response key were counterbalanced within subjects. The difference between the averaged response to antonym test words and that to synonym test words differed biphasically over the interval $250-650 \mathrm{msec}$ after the stimulus. The demonstration of an evoked response difference between synonyms and antonyms extends the applicability of evoked potentials from attributes of individual word meaning to the semantic relationships between words.
\end{abstract}

In several areas of cognitive research, investigators have adopted a multivariate approach in studying phenomena. For example, letter perception has been studied using accuracy, latency, effect on concurrent processing tasks, and evoked potentials (Posner, 1978). While this broad approach has been applied to many problems in psycholinguistics, only a little of this work has used the cortical evoked potential as a dependent measure. Recently, several attempts have been made to use the evoked potential as an indicator of the comprehension of meaning. While this research is new and at a pioneering stage, the results are promising. The present study attempted to determine whether evoked potentials were sensitive to certain semantic relationships more complex than those that have previously been studied. If it could be shown that evoked potentials reflect comprehension in tasks typically studied in semantic memory, then psycholinguistics will have another tool with which to investigate processes underlying the comprehension of meaning.

The average cortical evoked response has been shown

A preliminary report of this work was given at the 1981 meeting of the Eastern Psychological Association. The authors appreciate discussion with R.W. Chapman and J.W. McCrary, which helped to inspire the present study, and the comments of Frances Friedrich and Victor C. Vaughan. The work was supported by a grant to the first author from the Hamilton College Faculty Research Fund. The paper was completed while the first author was supported by National Institute of Mental Health National Research Service Award MH-08716 at the Psychology Department of the University of Oregon. R. L. O'Sullivan is now at the Neurological Research Laboratory, Jefferson Medical College. K. Sherif is now at University Hospital, University of Pennsylvania. Requests for reprints may be addressed to Jonathan Vaughan, Department of Psychology, Hamilton College, Clinton, New York 13323. by Chapman and his colleagues (Chapman, Bragdon, Chapman, \& McCrary, 1977) to reflect the connotation of words. This was done by presenting subjects with words that varied in connotative meaning (Glucksberg \& Danks, 1975) as measured by Osgood's semantic differential scale (Osgood, Suci, \& Tannenbaum, 1957). The semantic differential assesses words along three dimensions: evaluation ("good" or "bad"), activity ("active" or "passive"), and potency ("strong" or "weak"). When a test word was presented, the evoked response near the vertex was recorded for $1 \mathrm{sec}$. Chapman et al. (1977) measured the difference in evoked potentials between those words that fell high on the evaluative dimension ("good") and those that fell low on the dimension ("bad"). Difference templates were obtained from three subjects by taking the average evoked response as a function of time separately for "good" and "bad" items. These templates were then used to classify the evoked responses to evaluative words of nine other subjects into "good" and "bad" categories. Classification performance was significantly better than chance. This indicates that specific linguistic processes were evident in the evoked potentials. More recently, Chapman (1979) has reported a broader relationship between the shape of the evoked response and the semantic differential. He was able to determine at significantly greater than chance accuracy (by an objective, automated categorization of the evoked responses to each word) from which one of the six classes of the semantic differential the word presented on that trial was drawn.

The progress in discovering evoked potential correlates of meaning of individual words suggests that higher order semantic relationships, as are typically studied in semantic memory (Kintsch, 1980; Smith, 
1977), should be identifiable as well (e.g., relationships like antonymy and synonymity). The notion that two words might have the same meaning (that is, be synonymous) goes back at least as far as Aristotle. The concept that two words could be opposed in meaning (that is, be antonymous), although more modern, is also generally regarded in modern linguistics as one of the basic semantic relationships. The concepts of synonymity and antonymy have been of great interest to linguists and their latter-day colleagues, the psycholinguists, up to the present. Thus, the present research investigated whether evoked potentials reflect the processing differences that presumably must underlie semantic judgments regarding these two classic relationships.

It is of particular interest to psycholinguists to explore the brain mechanisms involved in the processing of higher order semantic relationships like synonymity and antonymy, because of the importance of these relationships in language. Synonym and antonym comprehension is a linguistic competence that is part of our everyday experience. Synonymity and antonymy are often believed to be opposite relationships; they are not. Rather, they are different both linguistically (Lyons, 1968) and psycholinguistically (Herrmann, Chaffin, Conti, Peters, \& Robbins, 1979). Synonymity is defined as the relationship between words that have the same denotative meaning. Antonymy is defined as the relationship between a pair of words that are opposed in meaning. The opposite of synonymity (sameness) is difference; the opposite of antonymy (opposition) is lack of opposition. Since these relationships figure so prominently in semantics and psycholinguistics, and differ from each other, they are primary candidates for research to determine whether the semantic relationships between pairs of words can be distinguished in observable electrical activity of the brain. However, previous studies that might have shown that evoked responses to synonyms are identifiably different from those to antonyms have not done so. Thatcher (1977) required subjects to determine whether a word was synonymous or antonymous with a previously presented word; no difference in evoked potential was reported for the type of relationship (see also Goto, Adachi, Utsonomiya, \& Chen, 1979).

The failure of previous studies (Goto et al., 1979; Thatcher, 1977) to detect evoked potential differences between synonym and antonym judgments may have been due to one or more of several reasons, such as the use of a small linguistic sample or insufficient counterbalancing to avoid idiosyncratic effects of stimulus words (cf. Clark, 1973). Thus, it might be expected that a larger sample of stimuli and more stringent linguistic controls would be necessary to permit observation of evoked potential differences between these semantic relationships. The present study used such improvements in procedure in an attempt to measure differences in evoked potentials for synonym and antonym judgments.

\section{METHOD}

\section{Subjects}

Five Hamilton College students were paid a minimum of $\$ 6$ for a $1.5 \mathrm{~h}$ session. Four were female (two left-handed) and one was male. Ages ranged from 20 to 22 years. Each subject's vision was $20 / 20$ or better (corrected, if necessary, with glasses) measured at a distance of $40 \mathrm{~cm}$ using an American Optical nearvision test card. (No subject was permitted to wear contact lenses during the experiment because the frequent blinks that the lenses often elicit cause large artifacts in the EEG record.) Each subject completed two counterbalanced sessions of about $1 \mathrm{~h}$ and $15 \mathrm{~min}$.

\section{Apparatus}

Electrode placement locations were scrubbed with isopropyl alcohol and electrodes (Grass ES-2) were applied with Grass EC2 electrode cream. The EEG was recorded from the scalp location CPz (Jasper, 1958), located on the midline $40 \%$ of the distance from the inion to the nasion. ${ }^{1}$ This is about $4 \mathrm{~cm}$ posterior to the vertex of the scalp, that is, the topmost part of the head. Reference electrodes on each earlobe were linked together. The resistance between $\mathrm{CPz}$ referenced to the linked ears was typically 3-5 kohms, and always less than 10 kohms. The signal was amplified by a Grass $P 15$ ac-coupled preamplifier (with bandpass of $1-\mathrm{Hz}$ to $100-\mathrm{Hz}$ half amplitude frequencies), further amplified by a Grass P18 dc preamplifier and, finally, recorded by a PDP-8 computer and stored for later analysis.

A second pair of electrodes was placed above and below the right eye to detect eye blinks and vertical eye movements. This electrooculogram (EOG) signal was also amplified with a Grass $\mathrm{P} 15$ preamplifier with a $1-\mathrm{Hz}$ to $100-\mathrm{Hz}$ bandpass. A temple electrode was used as ground for both preamplifiers.

\section{Procedure}

Stimulus presentation and evoked response recording. The subject was seated in a Faraday cage with his or her head resting on a cushioned headrest, eyes $43 \mathrm{~cm}$ in front of an oscilloscope screen, with the preferred hand resting on three response buttons (operated by the index, middle, and ring fingers, respectively). Before each trial, the word "READY" appeared at the center of the screen. When the subject initiated a trial (by pressing the middle button), a fixation point appeared for $500 \mathrm{msec}$, followed by the first word for $500 \mathrm{msec}$ displayed in uppercase, centered on the oscilloscope; each character subtended about $40 \times 30 \mathrm{~min}$ of visual angle. After a $1-\mathrm{sec}$ interword interval, the second word (the "test" word) was presented for $100 \mathrm{msec}$. The intensity of the stimuli was adjusted for comfortable viewing by a dark-adapted subject. The refresh rate of the display was $250 \mathrm{~Hz}$. In each trial, the EEG was sampled at $250 \mathrm{~Hz}$ for a period beginning $200 \mathrm{msec}$ before the second stimulus, for a total period of $2,000 \mathrm{msec}$, making 500 sample points/trial.

Manual choice response. The subject was instructed not to move or blink until a cue to respond was presented. During the sampling interval, and for the following 1 sec, the computer also integrated the rectified EOG signal; if this exceeded an adjustable threshold, the trial was canceled because of the likelihood of a blink or vertical eye movement artifact in the evoked response. The question "Antonym or Synonym?" was then displayed, beginning $2,000 \mathrm{msec}$ after the beginning of the sampling interval, as a cue for the subject to respond by pressing the left or right key. The possibility of response artifact in the evoked response was minimized by requiring all responses to be made by pressing the corresponding left or right button with the index or middle finger of the same (preferred) hand. So that the direction of the response (that is, left or right for synonym) would not be confounded with pair type, it was counterbalanced across sessions. Responseaccuracy feedback ("correct" or "incorrect") was shown to the subject after each trial, along with a cumulative point count from the beginning of the session. A monetary payoff 
for accuracy was used to maintain attention to the word pairs. Each correct response earned 1 point (worth 3 cents), and each error earned a fine of 10 points. Overall accuracy was greater than $99 \%$ correct. If the subject pushed a response button before the response cue appeared on the screen, the admonition "Don't anticipate!" was shown, and the trial was canceled.

Word list. The 256-pair word list presented to the subjects was divided into eight 32 pair blocks. The word pairs were taken from a list that had certain important qualities counterbalanced (see appendix). Within each category, the four words were combined into eight pairs so that every word appeared twice as the first and twice as the second (that is, test) word; each also appeared twice as a synonym and twice as an antonym. Thus, the average evoked response for synonyms resulted from exposure of the same set of test words in the same physical and temporal locations as the average evoked response for antonyms; only the context (the semantic relationship to the prior word of the pair) differed. Nearly all the words in the list were adjectives, and no pair differed only by a morphological marker (such as un-, anti-, or non-) that made antonymy explicit. One randomization of the list was used; a subject began each session at a different place within the list.

After approximately 10-20 practice trials (during which the adequacy of recording was verified), the room was darkened while the subject adapted to the dark for $7 \mathrm{~min}$. Between blocks of trials, the subject rested for at least $30 \mathrm{sec}$. After each session, the evoked responses of the individual trials were reviewed, and any that showed obvious artifacts, such as alpha waves or $60-\mathrm{Hz}$ interference, were excluded (this comprised $20 \%-52 \%$ of the trials). ${ }^{2}$ Finally, all the responses from correctly reported synonym trials were averaged together, and those of antonym trials were averaged together.

\section{RESULTS}

Figure 1 shows averaged evoked responses for synonyms and antonyms for two subjects. The figure shows the average voltage recorded at the scalp for the $200-\mathrm{msec}$ period before the presentation of the second word, the $100-\mathrm{msec}$ presentation time of the second word, and the $1,700 \mathrm{msec}$ following it. Figure 2 shows the corresponding average differences between the anto-

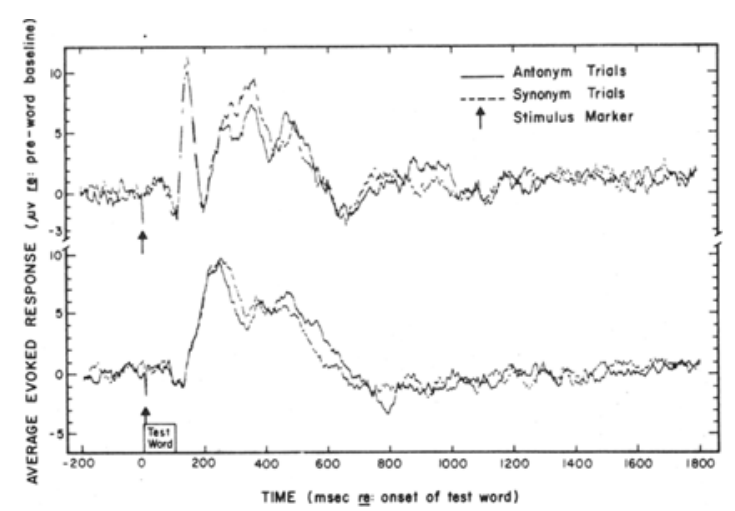

Figure 1. Evoked responses to the second word of each pair, separately for antonyms and synonyms, for two subjects. The recording begins $200 \mathrm{msec}$ before the onset of the test word and continues for a total of $2,000 \mathrm{msec}$. In this and the other figures, the line representing the evoked response is momentarily deflected (arrow) to the baseline at the onset of the test word.

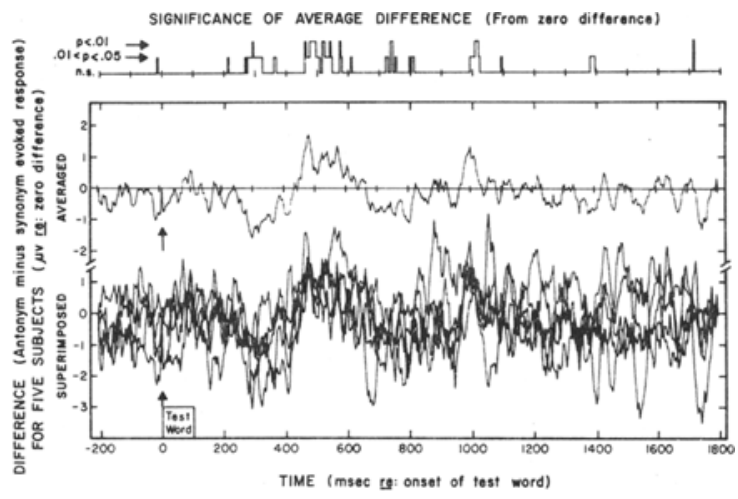

Figure 2. Bottom: Difference (antonym minus synonym) between the antonym and synonym evoked response at each point in the 2 -sec sampling interval, Five subjects' differences are superimposed. Middle: Average difference for all five subjects. Top: Significance of the average difference from zero at each sampling interval. The baseline represents no significant difference; the line takes one step up at points at which the average difference is significantly nonzero at the .05 level or beyond and two steps up at points at which the average difference is nonzero at the .01 level or beyond.

nym and synonym responses (1) superimposed for all five subjects and (2) for the five subjects combined. The curves in Figure 2 were calculated by taking the averaged evoked response for antonym trials at each of the 500 sampling points and subtracting the value of the averaged evoked response for synonyms at the corresponding points. The line at the top of Figure 2 represents the results of statistical evaluation of the difference at each of the 500 points. The average difference (over five subjects) at each of the 500 samples was compared, using a t test, with what would have been expected by chance (no difference). The line at the top of Figure 2 is displaced upward by 1 unit if for that point, $t$ is significantly different at the .05 level and by 2 units if it is significant at the .01 level. While these $t$ tests are not independent (since they involve the same subjects and consecutive points covary), the frequency and pattern of significance provide useful indexes of whether or not reliable differences in evoked potentials were obtained. ${ }^{3}$ The synonym response was greater than the antonym response (that is, the difference, antonym minus synonym, was negative) for the period $250400 \mathrm{msec}$ after the onset of the second stimulus, and the antonym response was greater than the synonym response for the period $450-650 \mathrm{msec}$ poststimulus. Comparison of the difference waveforms with the original data suggests that the peak of the early positive component of the averaged evoked response tends to be higher in synonym than in antonym trials and that the antonym evoked response is more positive, and remains positive longer, during the later positive component. As a result, the difference waveform (top half of Figure 2) is characterized by a biphasic difference, first negative (synonym trials greater than antonym) and then positive, over the time interval 


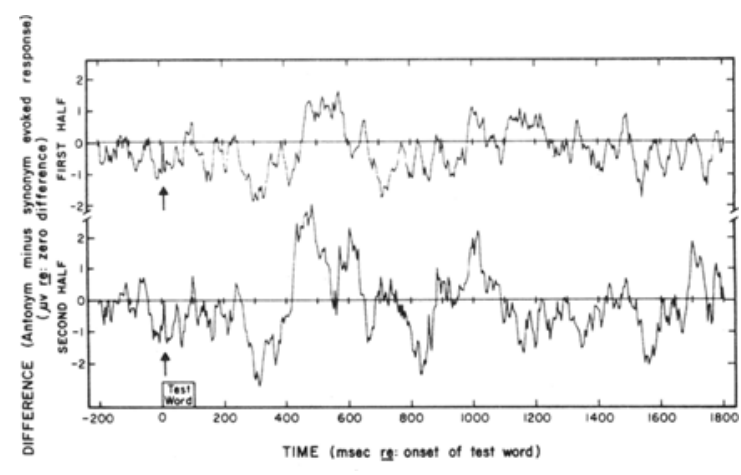

Figure 3. Mean difference between antonym and synonym evoked responses $(\mathrm{N}=5$ ) for (top) the combined first halves and (bottom) the second halves of the two sessions of five subjects.

$250-650 \mathrm{msec}$ after the onset of the stimulus. Both the first and second halves of the sessions show the same general pattern of difference (Figure 3).

\section{DISCUSSION}

The present findings establish that a difference between the evoked responses to synonyms and antonyms can be detected in the average of these responses. In so doing, this study extends the research applicability of evoked potentials from that found for the meaning responses of individual words (Chapman et al., 1977) to semantic relationships between words (Goto et al., 1979; Thatcher, 1977).

How should we interpret the observed differences in evoked responses for synonyms and antonyms? There are several physiological reasons that a difference in the averaged evoked response might come about. First, there may be a difference in the time course of cortical events correlated with the type of cognitive processing that is elicited by a particular semantic relationship. Consider, for example, the possibility that both synonyms and antonyms result in generally similar evoked responses, but that the process of recognizing antonyms is more variable in time than that of synonyms. Thus, some component of the response to antonyms could occur at more variable times. Then, when the responses to synonyms and antonyms were separately averaged, the average evoked response to antonyms would have a smaller amplitude, but a longer lasting deviation from the baseline; when this curve was compared by subtraction with the other, a biphasic response would be apparent in the resulting difference waveform. There is no clear evidence for this possibility in the present data; the peaks of the late positive potentials $(200-800 \mathrm{msec}$ poststimulus) for synonyms and antonyms do not appear to have consistently different latencies. Although judging one relation might well take longer than judging the other, current evidence is not sufficient for concluding which is the longer and whether the size of such a difference could account for the present results. Correct classification of synonyms was slower than that of antonyms in one study (Herrmann et al., 1979); however, this is but one considerably different study that did not counterbalance words between relationship pairs, as was done in the present study.

Second, we might consider the possibility that the response to one category is more vigorous, at the same temporal and spatial location, than the other. Alternatively, different cortical areas may be more active for each of the two semantic relationships considered here. In this case, the difference waveform would reflect the placement of the recording electrode over an area more nearly optimum for one type of relationship's evoked activity than for the other's. The present data do not permit us to distinguish among these alternative hypotheses of spatial or temporal differences in evoked responses. Suffice it to say that it is not possible to directly identify a particular peak of activity in the averaged evoked response as being "antonymy" or "synonymity" (see Chapman, McCrary, Bragdon, \& Chapman, 1979, for more extensive discussion of the problems of extracting and interpreting the components of waveform differences).

The linguistic basis of the present evoked potential difference may also be subjected to alternative explanations. For example, it is possible that the present evoked potential difference does not represent the full range of possible differences between these semantic relationships. Despite the structure of the tasks, it is possible that subjects might have reinterpreted the task to be one of detecting merely the presence or absence of antonyms, for example. If subjects did adopt such an approach, their response on each trial would need only be based on a single semantic criterion (e.g., the presence or absence of opposition); then, the difference waveform (Figure 2) would reflect the presence of this one attribute. An indication of whether or not subjects simplified their task in this manner may be obtained by inspecting data from other tasks in which same-different judgments are made. If subjects treated opposition as equivalent to difference, the waveform differences in same-different tasks should be similar in shape to those obtained here. Posner, Klein, Summers, and Buggie (1973) asked subjects to press a key to report "same" or "different" after presentation of a pair of letters. The average evoked responses differed such that the late positive response to matching stimuli was greater than that to mismatched ones, the difference having the same polarity as the first phase of the bipolar difference observed in the present study; the evoked response to "different" stimuli was more negative than that to "same" stimuli about $300 \mathrm{msec}$ after the onset of the second stimulus. In a very similar experiment with pairs of words, the direction of this monophasic difference could be reversed by requiring subjects to count only the 
"different" trials. The difference between "different" and "same" when "same" was attended to had a latency that was about $100 \mathrm{msec}$ shorter than that when "different" was attended to (Posner et al., 1973). The biphasic evoked response difference observed between synonyms and antonyms in the present study does not seem, then, to reflect anything as simple as mere detection of "difference" on antonym trials. On the other hand, the biphasic difference is possibly consistent with attention to both the "sameness" of synonyms and the "difference" of antonyms, if the responses to word pairs have a time course that is somewhat longer than that to single letters, as seems plausible. However, there is no consistent evidence of latency differences between synonym and antonym trials analogous to those observed by Posner et al. (1973), even in the clearest of the individual waveforms (Figure 1).

Alternatively, it is possible that the subjects used strict criteria of antonymy and synonymity: not just "difference," but true opposition to identify antonyms, and not just "sameness," but identity of denotation to identify synonyms. Resolution of which alternative is correct might be determined by using a task that would force subjects to consider most or all of the criteria of each relationship; for example, subjects might be asked to identify only the true synonyms and antonyms among pairs of items that contained pseudosynonym ("slow-late") and pseudoantonym ("popularshy") pairs as well (Herrmann et al., 1979). Such a task could not be solved by the mere detection of opposition.

The semantic explanation of the present differences in evoked responses to synonyms and antonyms is not, of course, the only possible one. First, different evoked responses may have originated because one relationship is harder to judge than another (the possibility of such a difference in difficulty does not exist for the stimulus words per se, since each word appeared in both relationship judgments). However, no previous work has clearly shown synonym judgments to be harder than antonym judgments, as measured by reaction time (Chaffin \& Herrmann, 1981; Herrmann, 1978; Herrmann et al., 1979). Second, the present differences may have resulted from disparities in confidence between the two relationship judgments. Again, however, no study has asked subjects to make confidence ratings for synonym and antonym judgments (or other semantic judgments). It is an open question whether confidence might differ between the judgments of these relationships. As for the present study, no subjects volunteered confidence statements (e.g., that one relationship was easier than another). Third, even if difficulty, decision speed, and confidence were equal between synonym and antonym judgments, the relationships might differ in how much they invoke processing of real-world knowledge (interchangeability in sentences or empirical strength of association, for example) rather than more abstract semantic properties per se. However, there have been no studies reported that have disentangled these two aspects of meaning in relationship judgments (see Chaffin, 1979). Fourth, a difference in the average evoked response for synonyms and antonyms could arise indirectly from some other effect. One mechanism by which this could occur depends on the tendency of the endogenous P300 component of the averaged evoked response (a positive deflection of the evoked response that occurs $300-600 \mathrm{msec}$ after many stimuli) to be larger when relatively unexpected stimuli are presented to a subject (Horst, Johnson, \& Donchin, 1980). Now, if subjects were to adopt a strategy of generating an antonym of the first word on each trial, then that expectation would be violated on synonym trials. Thus, on synonym trials, the evoked response would be larger than on antonym trials, in which the "expected" test word occurs. ${ }^{4}$ However, as subjects developed familiarity with the words used in this experiment, we might predict the effect of such a priori expectations to be attenuated. To the contrary, the difference between synonym and antonym judgments was at least as large in the second halves of the sessions as in the first (Figure 3).

Thus, there are several potential explanations of the present results. Past research gives us few clues as to which explanation or explanations will prove appropriate. Nevertheless, all of these explanations appear tractable to investigation. Until we obtain evidence to the contrary, we hypothesize that the semantic difference between synonyms and antonyms is a viable explanation of the observed difference in evoked responses.

Several areas of further study promise to be productive. First, Thatcher (1977) has shown some difference between the two hemispheres in the response to semantic stimuli. Understanding of the processing bases of the present difference might well come from explorations of the locus of maximal difference between semantic relationships. Second, it is useful to consider the linguistic variables that may influence evoked potentials. One of these may be the characteristics of the particular stimuli used. It has been shown that the reaction time to identify a synonym or antonym pair varies with the judged "goodness" of the relationship (Herrmann, 1978; Herrmann et al., 1979). Some aspects of evoked responses vary with reaction time. For example, Kutas, McCarthy, and Donchin (1977) have shown that the late positive component (P300) of the averaged evoked response is related to reaction time for the identification of words of a particular class: The longer the latency of P300, the longer is the reaction time, especially when the subjects are instructed to respond with a set for accuracy. A comparison of "good" with "poor" synonyms would be of interest in the present paradigm. Regardless of the specific direction of future research, it is clear that it is possible to measure the evoked potential correlates of semantic relationships. 


\section{REFERENCES}

Chaffin, R. Knowledge of language and knowledge about the world: A reaction-time study of invited and necessary inferences. Cognitive Science, 1979, 3, 311-328.

Chafpin, R., \& Herrmann, D. J. Comprehension of semantic relationships and the generality of categorization models. Bulletin of the Psychonomic Society, 1981, 17, 69-72.

Chapman, R. M. Connotative meaning and averaged evoked potentials. In H. Begleiter (Ed.), Evoked brain potentials and behavior. New York: Plenum, 1979.

Chapman, R. M., Bragdon, H. R., Chapman, J. A., \& McCrary, J. W. Semantic meaning of words and averaged evoked potentials. Progress in Clinical Neurophysiology, 1977, 3, 36-47.

Chapman, R. M., McCrary, J. W., Bragdon, H. R., \& Chapman, J. A. Latent components of event-related potentials functionally related to information processing. Progress in Clinical Neurophysiology, 1979, 6, 80-105.

Clark, H. H. The language-as-fixed-effect fallacy: A critique of language statistics in psychological research. Journal of Verbal Learning and Verbal Behavior, 1973, 12, 335-359.

Guucksererg, S., \& DANks, J. H. Experimental psycholinguistics. Hillsdale, N.J: Erlbaum, 1975.

Goto, H., Adachi, T., Utsonomiya, T., \& Chen, I. C. Late positive component (LPC) and CNV during processing of linguistic information. In D. Lehmann \& E. Callaway (Eds.), Human evoked potentials. New York: Plenum, 1979.

HerrmanN, D. J. An old problem for the new psychosemantics: Synonymity. Psychological Bulletin, 1978, 85, 490-512.

Herrmann, D. J., Chaffin, R. J. S., Conti, G., Peters, D., \& Robrins, P. H. Comprehension of antonymy and the generality of categorization models. Journal of Experimental Psychology: Human Learning and Memory, 1979, 5, 585-597.

Honst, R. L., Johnson, R., \& Donchin, E. Event-related brain potentials and subjective probability in a learning task. Memory \& Cognition, 1980, 8, 476-488.

JASPER, H. H. The ten-twenty electrode system of the international federation. Electroencephalography and Clinical Neurophysiology, 1958, 10, 371-375.

Jones, K. G., \& Armington, J. C. The removal of alpha from the VECP by means of selective averaging. Vision Research, $1977,17,949-956$.

KINTSCH, W. Semantic memory: A tutorial. In R. S. Nickerson (Ed.), Attention and performance VIII. Hillsdale, N.J: Erlbaum, 1980.

Kutas, M., \& Hillyard, S. A. Reading senseless sentences: Brain potentials reflect semantic incongruity. Science, 1980 , 207, 203-205.

Kutas, M., McCarthy, G., \& Donchin, E. Augmenting mental chronometry: The P300 as a measure of stimulus evaluation time. Science, 1977, 197, 792-795.

Lansing, R. W., Schwartz, E., \& Lindsley, D. B. Reaction time and EEG activation under alerted and nonalerted conditions. Journal of Experimental Psychology, 1959, 58, 1-7.

LyONS, J. Introduction to theoretical linguistics. Cambridge, England: Cambridge University Press, 1968.

Osgood, C. E., Sucr, G. J., \& Tannenbaum, P. The measurement of meaning. Urbana: University of Illinois Press, 1957.

Posner, M. I. Chronometric explorations of mind. New York: Erlbaum, 1978.

Posner, M. I., Klein, R., Summers, J., \& Buggie, S. On the selection of signals. Memory \& Cognition, 1973, 1, 2-12.

SMITH, E. E. Theories of semantic memory. In W. K. Estes (Ed.), Handbook of learning and cognitive processes (Vol. 5). Hillsdale, N.J: Erlbaum, 1977.

Thatcher, R. W. Evoked potential correlates of hemispheric lateralization during semantic information processing. In $S$. Harrad, R. W. Doty, L. Goldstein, J. Jaynes, \& G. Krauthamer (Eds.), Lateralization in the nervous system. New York: Academic Press, 1977.
Webster's Third New International Dictionary. Springfield, Mass: Merriam, 1971.

\section{NOTES}

1. By convention, the placement of EEG electrodes is relative to reproducible landmarks on the skull (Jasper, 1958). The inion is the occipital protuberance of the skull; the nasion is the most indented point of the bridge of the nose (see Webster's Third New International Dictionary, 1971, entry for "craniometry"). The path along the midline of the scalp between these two reference points is used to establish electrode placements that are reproducible between and within subjects. The vertex (now usually labeled $\mathrm{Cz}$ ) is $50 \%$ of the distance from inion to nasion; the recording site used here, $\mathrm{CPz}$, is slightly posterior, at $40 \%$ of the inion-nasion distance. This is about $1 \mathrm{~cm}$ posterior to the location used in the studies of Chapman and his colleagues (Chapman, 1979; Chapman et al., 1977), discussed in the introduction.

2. Each trial was displayed on an oscilloscope screen for inspection for artifacts; this was done blind to the stimulus item presented on that trial. Most excluded trials were contaminated with alpha (high-amplitude $8-$ to $14-\mathrm{Hz}$ ) activity; Jones and Armington (1977) have shown that averaged evoked responses are clearer if these trials are excluded. The rationale for excluding these trials included the fact that alpha is correlated with relative inattention; for instance, it is "blocked" when subjects are solving mental arithmetic problems. A trial with alpha activity may represent suboptimal attention to the task on the part of the subject (Lansing, Schwartz, \& Lindsley, 1959), and its inclusion could attenuate effects of other variables in an experiment.

A second reason for discarding alpha trials and those with apparent movement artifacts is that these would be likely to have a disproportionate effect on the final averages. The theoretical rationale for the use of signal averaging techniques assumes that noise is temporally uncorrelated with the evoked response of interest and, further, that it occurs with random amplitude so that it will average to zero across trials. Neither of these two assumptions holds for movement artifacts: They occur (with large amplitude) on only a few trials, and they are probably temporally correlated with the response of interest, since they are caused by an anticipatory manual response that is not large enough to close the response switch. It is possible that neither of the two assumptions holds in the case of alpha, either. Alpha may be driven in synchrony with repeated visual stimulation in the 8- to 12-Hz range in some subjects; in the present paradigm, the brightening of the visual field at stimulus onset might serve to synchronize alpha activity from trial to trial so that it would not average to zero.

After exclusion of error and artifact trials, there remain in the averaged data from 244 to 323 trials/subject (median $=272)$ of the original 512 trials.

3. There are two related reasons that these statistics are not independent. First, the comparison at each sampling interval involves the same five subjects. Second, since these are timeseries data, if the difference at a single sampling interval is significant by chance, the likelihood of the next difference also being significant is higher than the nominal alpha level. With this reservation in mind, we may look at whether the number of significant points is within chance expectations. If we were to assume independence, we would expect to observe by chance (in 200 $t$ tests on the differences occurring in the $800 \mathrm{msec}$ after the beginning of the second stimulus word) 10 significant comparisons at the .05 level and 2 at the .01 level. We actually observed 45 at the .05 level and 13 at the .01 level, respectively, markedly above that expectation; these are clustered together during $200-650 \mathrm{msec}$ after the stimulus, rather than being more uniformly distributed. As a partial control for the nonindependence of these tests, let us take the $1,000 \mathrm{msec}$ beginning $800 \mathrm{msec}$ after stimulus presentation, a period when no evoked response was expected to occur. During this interval, we observed that in 
only 16 samples was the difference between antonym and synonym evoked responses significantly different from zero at the .05 level, and 4 were significant at the .01 level, very nearly the numbers that would be expected (12.5 and 2.5 , respectively) assuming independence. The binomial test for the difference between two frequencies indicates that the 61 points significant at the .05 level are not randomly distributed between the 800 -msec evoked response interval and the $1,000-\mathrm{msec}$ "control" interval $\left(\mathrm{z}=5.28, \mathrm{p}<10^{-6}\right)$. This comparison, although numerically impressive, must also be interpreted in light of the uncertain independence of differences at consecutive sampling intervals.

4. A similar argument could be proposed for comparing the present results to the "incongruity" potential (N400) reported by Kutas and Hillyard (1980) for words that are semantically incongruous with the sentence in which they appear (e.g., "socks" in the sentence "He spread the warm bread with socks."). The problem with drawing this analogy is that it is not at all clear what is "incongruous" in the present comparison of synonym and antonym pairs. One could argue either way: that an antonym is incongruous (since it differs in meaning from the first word of the pair) or that a synonym is incongruous (when the first word is seen, it might be easier to generate and expect the unique best antonym of that word than to generate and expect the particular synonym used in the experiment from among the several plausible candidates that usually exist).

(Received for publication May 1, 1981; revision accepted January 18,1982 .)

Appendix

Word List Used in the Experiment

\begin{tabular}{|c|c|c|c|c|}
\hline \multicolumn{5}{|c|}{ Example } \\
\hline \multicolumn{2}{|r|}{ Synonyms } & & \multicolumn{2}{|c|}{ Antonyms } \\
\hline \multicolumn{2}{|c|}{ First Word } & Test Word & First Word & Test Word \\
\hline \multirow{4}{*}{\multicolumn{2}{|c|}{$\begin{array}{l}\text { BIG } \\
\text { SMALL } \\
\text { LARGE } \\
\text { TINY }\end{array}$}} & \multirow{4}{*}{$\begin{array}{l}\text { LARGE } \\
\text { TINY } \\
\text { BIG } \\
\text { SMALL }\end{array}$} & \multirow{4}{*}{$\begin{array}{l}\text { BIG } \\
\text { SMALL } \\
\text { LARGE } \\
\text { TINY } \\
\end{array}$} & SMALL \\
\hline & & & & $\mathrm{BIG}$ \\
\hline & & & & TINY \\
\hline & & & & LARGE \\
\hline Category & Word A & Word B & Word C & Word D \\
\hline 1 & UPTIGHT & TENSE & RELAXED & CALM \\
\hline 2 & MISSING & ABSENT & PRESENT & HERE \\
\hline \multirow{2}{*}{$\begin{array}{l}3 \\
4\end{array}$} & PERMIT & ALLOW & PREVENT & THWART \\
\hline & ILL & SICK & HEALTHY & WELL \\
\hline 5 & BARREN & STERILE & FERTILE & PRODUCTIVE \\
\hline 6 & PERMANENT & ETERNAL & TEMPORARY & TRANSIENT \\
\hline 7 & HIDDEN & CONCEALED & EXPOSED & VISIBLE \\
\hline \multirow{2}{*}{$\begin{array}{l}8 \\
9\end{array}$} & LIFELESS & DEAD & ALIVE & ANIMATED \\
\hline & ACCURATE & TRUE & FALSE & FALLACIOUS \\
\hline 10 & CONFIRM & VERIFY & REFUTE & REBUT \\
\hline 11 & BLAMELESS & INNOCENT & GUILTY & CULPABLE \\
\hline \multirow{2}{*}{$\begin{array}{l}12 \\
13\end{array}$} & REQUIRED & COMPULSORY & VOLUNTARY & OPTIONAL \\
\hline & SURE & CERTAIN & DOUBTFUL & UNDECIDED \\
\hline 14 & CONTINUE & PERSIST & STOP & CEASE \\
\hline 15 & MINDFUL & ATTENTIVE & CARELESS & NEGLIGENT \\
\hline \multirow{2}{*}{16} & BIG & LARGE & SMALL & TINY \\
\hline & ELEMENTARY & SIMPLE & COMPLEX & INTRICATE \\
\hline $\begin{array}{l}17 \\
18\end{array}$ & RIGHT & CORRECT & WRONG & MIST AKEN \\
\hline 19 & LUCID & CLEAR & FOGGY & CLOUDY \\
\hline 20 & TANGIBLE & CONCRETE & ABSTRACT & THEORETICAL \\
\hline 21 & PROFOUND & DEEP & SHALLOW & SUPERFICIAL \\
\hline 22 & USUAL & COMMON & ECCENTRIC & ATYPICAL \\
\hline 23 & CHAOS & ANARCHY & HARMONY & ORDER \\
\hline 24 & LOVELY & BEAUTIFUL & UGLY & UNSIGHTLY \\
\hline 25 & YOUNG & YOUTHFUL & OLD & AGED \\
\hline 26 & PREVIOUS & PAST & FUTURE & HEREAFTER \\
\hline 27 & SPIRITED & ACTIVE & PASSIVE & LISTLESS \\
\hline 28 & HAPPY & GLAD & SAD & MELANCHOLY \\
\hline 29 & VICTORY & TRIUMPH & DEFEAT & DOWNFALL \\
\hline 30 & THIN & SLIGHT & WEIGHTY & HEFTY \\
\hline 31 & DESPISE & HATE & LOVE & ADORE \\
\hline 32 & TRIFLING & TRIVIAL & IMPORTANT & SIGNIFICANT \\
\hline
\end{tabular}

Note-Each category consists of four words that were combined to make eight pairs, as shown in the example for Category 16. Note that each word appears equally often in the first position followed by a synonym or antonym and each appears in the second position equally often in the rale of a synonym and in that of an antonym. In each session, the subject viewed the list of 256 pairs of words in random order, with a different starting place in the list. For example, the four words of Category 16 generated a total of four pairs in which the test words were synonyms and four in which the test words were antonyms of the first word of the pair. 